\title{
Chronic inflammation-related DNA damage response: a driving force of gastric cardia carcinogenesis
}

\author{
Runhua Lin ${ }^{1,2, *}$, Dejun Xiao ${ }^{1,4, *}$, Yi Guo ${ }^{3}$, Dongping Tian ${ }^{1,2}$, Hailong Yun ${ }^{1,2}$, Donglin \\ Chen $^{1,2}$ and Min Su ${ }^{1,2}$ \\ ${ }^{1}$ Institute of Clinical Pathology, Guangdong Provincial Key Laboratory of Infectious Disease and Molecular Immunopathology, \\ Shantou University Medical College, Shantou, Guangdong, PR China \\ 2 The Judicial Critical Center, Shantou University Medical College, Shantou, Guangdong, PR China \\ ${ }^{3}$ Cancer Hospital of Shantou University Medical College, Shantou, Guangdong, PR China \\ ${ }^{4}$ Clinical Laboratory of Ganzhou People's Hospital, Ganzhou, Jiangxi, PR China \\ * These authors contributed equally to this work
}

Correspondence to: Min Su, email: minsu@stu.edu.cn

Keywords: chronic inflammation, DNA damage response, precancerous lesions, genomic instability, gastric cardia carcinogenesis Received: November 10,2014 Accepted: December 25, $2014 \quad$ Published: December 30, 2014

This is an open-access article distributed under the terms of the Creative Commons Attribution License, which permits unrestricted use, distribution, and reproduction in any medium, provided the original author and source are credited.

\section{ABSTRACT}

Gastric cardia cancer (GCC) is a highly aggressive disease associated with chronic inflammation. To investigate the relationship between DNA damage response (DDR) and chronic inflammation, we collected 100 non-tumor gastric cardia specimens of Chaoshan littoral, a high-risk region for esophageal and gastric cardia cancer. A significantly higher proportion of severe chronic inflammation was found in dysplastic epithelia $(80.9 \%)$ in comparison with that in non-dysplastic tissues $(40.7 \%)(P<0.001)$. Immunohistochemical analysis demonstrated that DNA damage response was parallel with the chronic inflammation degrees from normal to severe inflammation $(P<0.05)$. We found that DNA damage response was progressively increased with the progression of precancerous lesions $(P<0.05)$. These findings provide pathological evidence that persistent chronic inflammation-related DNA damage response may be a driving force of gastric cardia carcinogenesis. Based on these findings, DNA damage response in non-malignant tissues may become a promising biomedical marker for predicting malignant transformation in the gastric cardia.

\section{INTRODUCTION}

Gastric cancer represents the fourth most common cancer and second leading cause of cancer-related death worldwide, taking a toll of approximately 738,000 people in 2008 alone [1]. It was once considered as a single entity. Now, scientists have divided this cancer into two main types: gastric cardia cancer (cancer occurs in the top portion of the stomach near the gastro-esophageal junction) and non-cardia gastric cancer (cancer in all other areas of the stomach). The incidence of GCC has a remarkable geographic aggregation, which resembled the epidemiological features of esophageal squamous cell carcinoma (ESCC), particularly in high-risk areas $[2,3]$. Our previous epidemiological study showed a high incidence of GCC $(34.8 / 100,000)$ in Nan'ao Island of Shantou, an isolated Chaoshan littoral region of China, from 1995 to 2004 [2], highlighting the need for greater understanding of its pathogenesis. Nevertheless, the vast majority of cancers originating from gastric cardia are adenocarcinomas based on histopathological features. These epidemiological and histopathological characteristics confer uniqueness to GCC as a clinical entity. The gastro-esophageal junction (including the proximal gastric cardia region) is an anatomical location with a remarkably high and rapidly rising incidence of adenocarcinoma [4-6], while the underlying mechanisms for the development of GCC are poorly understood [7].

Inflammation is a vital defensive response that serves as critical roles in a variety of physiological situations, and when dysregulated, can contribute to the 
Table 1: Summary of the baseline data of GCC patients and the examined

\begin{tabular}{ll}
\multicolumn{1}{c}{ Vamples } & \multicolumn{1}{c}{ No. } \\
\hline Age, yrs (range), $\mathrm{n}=50$ & $62.3 \pm 8.1(46-76)$ \\
Sex, $\mathrm{n}=50$ & $38(76)$ \\
Male (\%) & $12(24)$ \\
Female (\%) & \\
Histological condition, $\mathrm{n}=100$ & $47(47)$ \\
Normal/hyperplasia (\%) & $48(48)$ \\
LGIN (\%) & $5(5)$ \\
HGIN (\%) & \\
Chronic inflammation, $\mathrm{n}=100$ & $23(23)$ \\
Normal (\%) & $30(30)$ \\
Mild (\%) & $25(25)$ \\
Moderate (\%) & $22(22)$ \\
Severe (\%) & \\
\hline
\end{tabular}

LGIN: low-grade intraepithelial neoplasia; HGIN: high-grade intraepithelial neoplasia.

pathogenesis of many diseases, including cancer. Chronic inflammation is a well-documented risk factor for cancer development [8-10]. Links between inflammation and cancer were first reported by Rudolf Virchow in 1863, on the basis of observations that tumors often arise at sites of chronic inflammation and inflammatory cells were present in biopsy samples from tumors [11]. Later on, a substantial body of evidence revealed that chronic inflammation may be a causative factor of a variety of cancers. Clinical epidemiological studies revealed that over $15 \%-20 \%$ of all solid tumors may be related to chronic inflammation [12-15]. The question of how chronic inflammation can initiate tumorigenesis has interested scientists for decades and is far to be resolved and clarified. Therefore, there is a need for investigation on relevant mechanisms of chronic inflammation-related tumor development.

DNA damage occurs through exposure to various toxic agents, including inflammatory cytokines $[16,17]$. Under chronic inflammatory conditions, reactive oxygen species (ROS) and reactive nitrogen species (RNS) are generated and result in DNA damage [18]. Based on the results from our previous study regarding DNA damage response in peritumoral regions of esophageal cancer microenvironment, herein, we focus on the possible role of chronic inflammation-related DNA damage response during the process of gastric cardia carcinogenesis.

Today, the causal relationship between chronic inflammation and cancer is more widely accepted, whereas the underlying molecular mechanisms mediating this relationship continue to be elucidated. In this current study, we therefore investigate the relationship between DNA damage response status and chronic inflammation as well as intraepithelial neoplasia of the gastric cardia. Our findings showed that DNA damage response in non-malignant tissues correlated with enhanced chronic inflammation and was also associated with progression of precancerous lesions, suggesting a pivotal role of chronic inflammation-related DNA damage response in gastric cardia tumorigenesis.

\section{RESULTS}

\section{Significantly higher proportion of severe chronic inflammation in dysplastic tissues}

To clarify the possible role of chronic inflammation in gastric cardia carcinogenesis, it is of fundamental importance to evaluate chronic inflammation status in gastric cardia tissues without malignant changes. To achieve this, we examined a total of 100 non-malignant gastric cardia tissues (50 tumor-proximal non-malignant tissues and 50 distant non-malignant tissues) from 50 GCC patients by analyzing inflammation status in tissues with different pathological changes. Our results revealed that severe chronic inflammation was more frequently observed in dysplastic tissues (80.9\%), such as low-grade intraepithelial neoplasia (LGIN) and highgrade intraepithelial neoplasia (HGIN) in comparison with that in non-dysplastic (normal/hyperplasia) tissues $(40.7 \%)(\mathrm{P}<0.001)$ (Figure 1b-d), indicating a potential link between chronic inflammation and gastric cardia carcinogenesis.

\section{DDR is parallel with the severity of chronic inflammation}

It has been previously reported that the formation of DNA lesions induced by reactive oxygen and nitrogen species (RONS) during chronic inflammation could lead to colonic tumorigenesis in mice [17]. In addition, 
our previous study has demonstrated that DNA damage response was increased in esophageal cancer tissues and tumor-surrounding non-malignant tissues compared with more distant tissues in human esophageal tissues [19]. Hence, we speculated that whether DNA damage serves as the intermediate to bridge the gap between chronic inflammation and gastric cardia carcinogenesis. If that is the case, DNA damage response should be more likely to be detected in inflamed tissues than tissues without evident inflammation. First, we attempted to investigate the actual status of DDR in the above tissues with various degrees of chronic inflammation. Phosphorylation of histone $\mathrm{H} 2 \mathrm{AX}(\gamma \mathrm{H} 2 \mathrm{AX})$ and its recruitment are one of the very first events in indentifying damaged DNA, which is also a reliable marker for DNA double-strand breaks [20]. Not surprisingly, immunohistochemical analysis showed that different proportion of epithelial cells in inflamed tissues stained positive for $\gamma \mathrm{H} 2 \mathrm{AX}$. The strongest and most abundant immunostaining of $\gamma \mathrm{H} 2 \mathrm{AX}$ was observed in epithelial cells accompanied by severe chronic inflammation. A less intense $\gamma \mathrm{H} 2 \mathrm{AX}$ signal was found in samples with mild-to-moderate inflammation, normal gastric cardia tissues without evident chronic inflammation were totally negative for $\gamma \mathrm{H} 2 \mathrm{AX}$ (Figure 2a). Intriguingly, quantification analysis for $\gamma \mathrm{H} 2 \mathrm{AX}$ revealed a significant increase of $\gamma \mathrm{H} 2 \mathrm{AX}$ staining in tissues with severer chronic inflammation compared to samples without evident inflammation (Figure 2b). The same results were obtained by western blot analysis of $\gamma \mathrm{H} 2 \mathrm{AX}$ in these tissues (Figure 2c). To further ascertain this phenomenon, we subsequently sought to measure other DDR markers. The activation of ataxia telangiectasia mutated kinase (ATM) by phosphorylation at Ser1981 is an early event at DNA damage loci as well. Consistent with the immunohistochemical results of $\gamma \mathrm{H} 2 \mathrm{AX}$, phosphorylation of ATM was also detected in inflamed tissues, with stronger signal in severer degrees of chronic inflammation compared with non-inflammation tissues (Figure 2d). These results suggested that severe chronic inflammation, to some extent, was associated with higher levels of DDR in gastric cardia epithelia. Conforming to our hypothesis, elevated levels of DDR might be induced
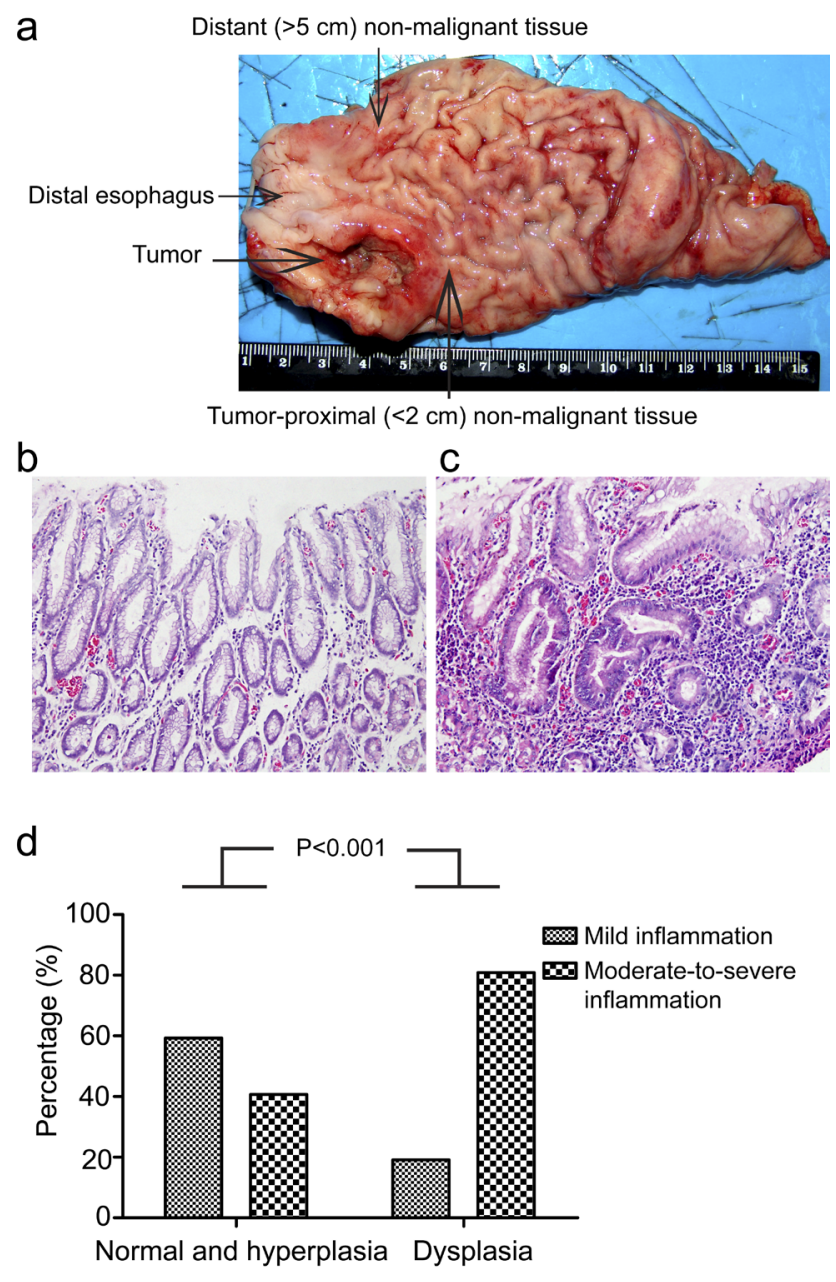

Figure 1: Chronic inflammation correlates with dysplasia in gastric cardia epithelia. (a) Schematic illustration of gastric cardia epithelial tissue sample collection. (b) Representative image of normal gastric cardia mucosa with mild inflammation. (c) Representative image of dysplastic gastric cardia mucosa with severe inflammation. (d) Percentage of different degrees of chronic inflammation in indicated types of tissues are shown. 
by chronic inflammation, which eventually precipitate carcinogenesis through genomic instability.

\section{DDR correlates with premalignant lesions}

We have now unveiled the close relationship between DNA damage response and chronic inflammation. It is reasonable to hypothesize that DDR is supposed to be evident in samples with precancerous lesions in case of its participation in tumor development. It is widely recognized that chronic inflammation of the gastric mucosa can progress through sequential premalignant stages from atrophic gastritis, intestinal metaplasia (IM) and dysplasia to ultimately gastric cancer [21, 22]. It is also well-known that the development of cancer is a complicated multi-step process involving multiple genetic alterations. In fact, our previous study has confirmed that DDR levels correlated significantly with the progression stages of premalignant lesions in esophageal squamous epithelium. To test our hypothesis, we analyzed the immunohistochemical results of DDR grouped by different pathological changes in gastric cardia tissues, such as histologically-normal epithelium, low-grade intraepithelial neoplasia (LGIN), and high-grade intraepithelial neoplasia (HGIN). Not surprisingly, immunohistochemical analysis showed that the expression of $\gamma \mathrm{H} 2 \mathrm{AX}$ progressively increased in the sequential stages from histologically-normal epithelia to HGIN (Figure 3a\&b), indicating increased genomic instability during the multi-step process of gastric cardia carcinogenesis. Additionally, we have confirmed the colocalization of $\gamma \mathrm{H} 2 \mathrm{AX}$ and p-ATM through performing double immunostaining in the same section (Figure 3c). These results suggested that unrepaired DNA damage in these precancerous gastric cardia tissues may be the source of its carcinogenesis.

\section{DISCUSSION}

In this study, we used human gastric cardia tissues to investigate the association between DNA damage response and chronic inflammation as well as precancerous lesions. Firstly, we observed that dysplastic epithelial cells of the gastric cardia were frequently accompanied by severe chronic inflammation characterized by macrophage and lymphocyte infiltration, prompting us to seek the underlying mechanisms bridging the gap between chronic inflammation and gastric cardia carcinogenesis.

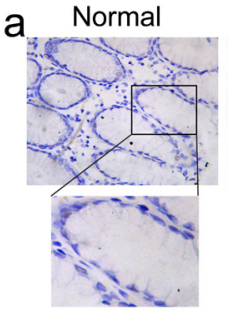

b

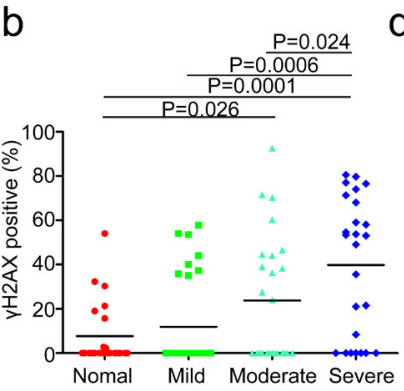

\section{Mild}

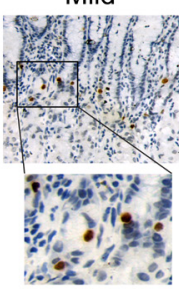

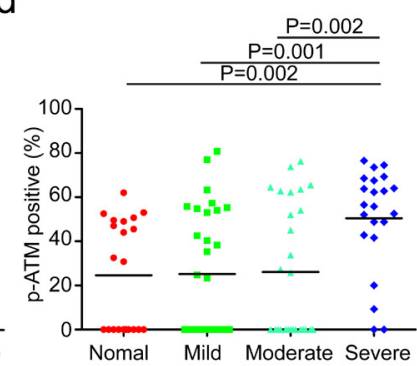

C

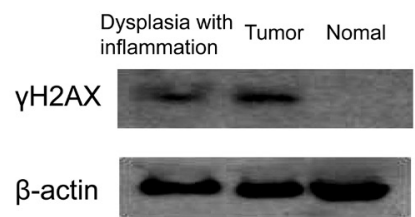

Figure 2: DNA damage response is associated with the degree of chronic inflammation. (a) Representative images of $\gamma \mathrm{H} 2 \mathrm{AX}$ staining in tissues without evident inflammation, with mild, moderate and severe inflammation. (b) Quantification of $\gamma \mathrm{H} 2 \mathrm{AX}$ positive cells in tissues without evident inflammation, with mild, moderate and severe inflammation from 50 gastric cardia cancer patients (two non-malignant samples from each patient). (c) Representative western blot analysis of $\gamma \mathrm{H} 2 \mathrm{AX}$ and ACTIN in tumor tissue, inflamed tissue with dysplasia and histologically normal tissue without evident inflammation. (d) Quantification of phospho-ATM positive cells in tissues without evident inflammation, with mild, moderate and severe inflammation from 50 gastric cardia cancer patients (two non-malignant samples from each patient). 


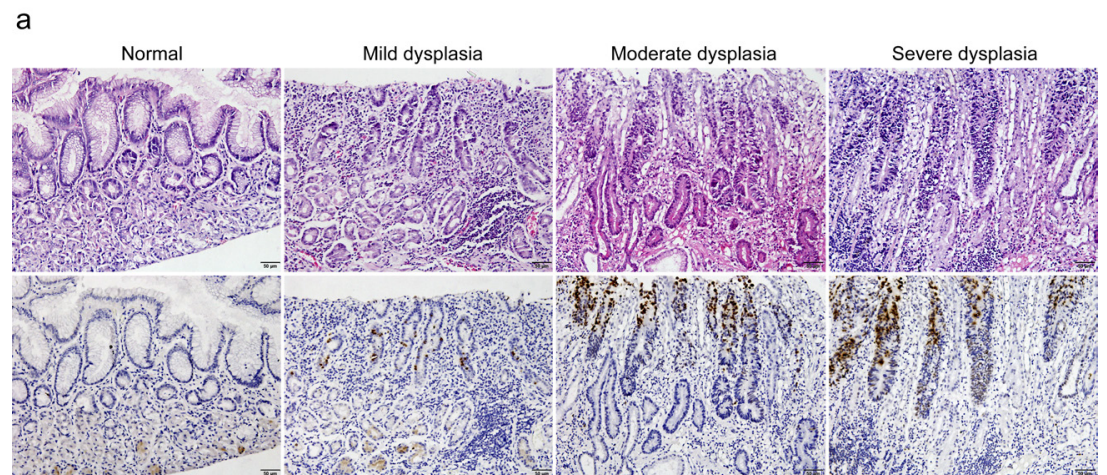

$\mathrm{b}$

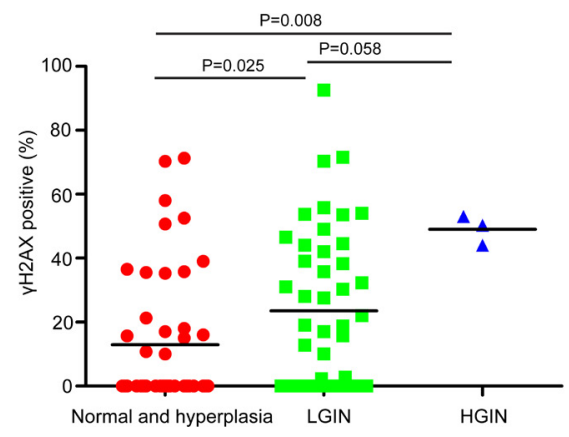

C

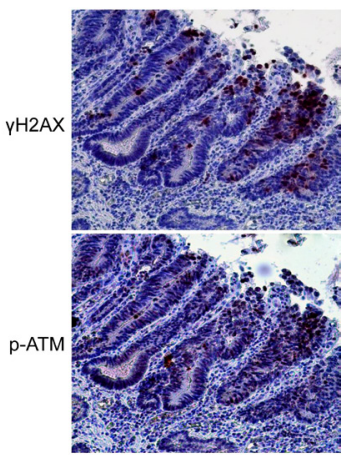

Figure 3: DNA damage response correlates with premalignant lesions in gastric cardia epithelia. (a) Representative images of $\gamma \mathrm{H} 2 \mathrm{AX}$ staining in normal, mild, moderate, and severe dysplastic tissues. (b) Quantification of $\gamma \mathrm{H} 2 \mathrm{AX}$ positive cells in non-malignant samples grouped according to benign or preneoplastic conditions. (c) Representative images showing the co-localization of $\gamma \mathrm{H} 2 \mathrm{AX}$ and phospho-ATM in dysplastic tissues with chronic inflammation in the same section.
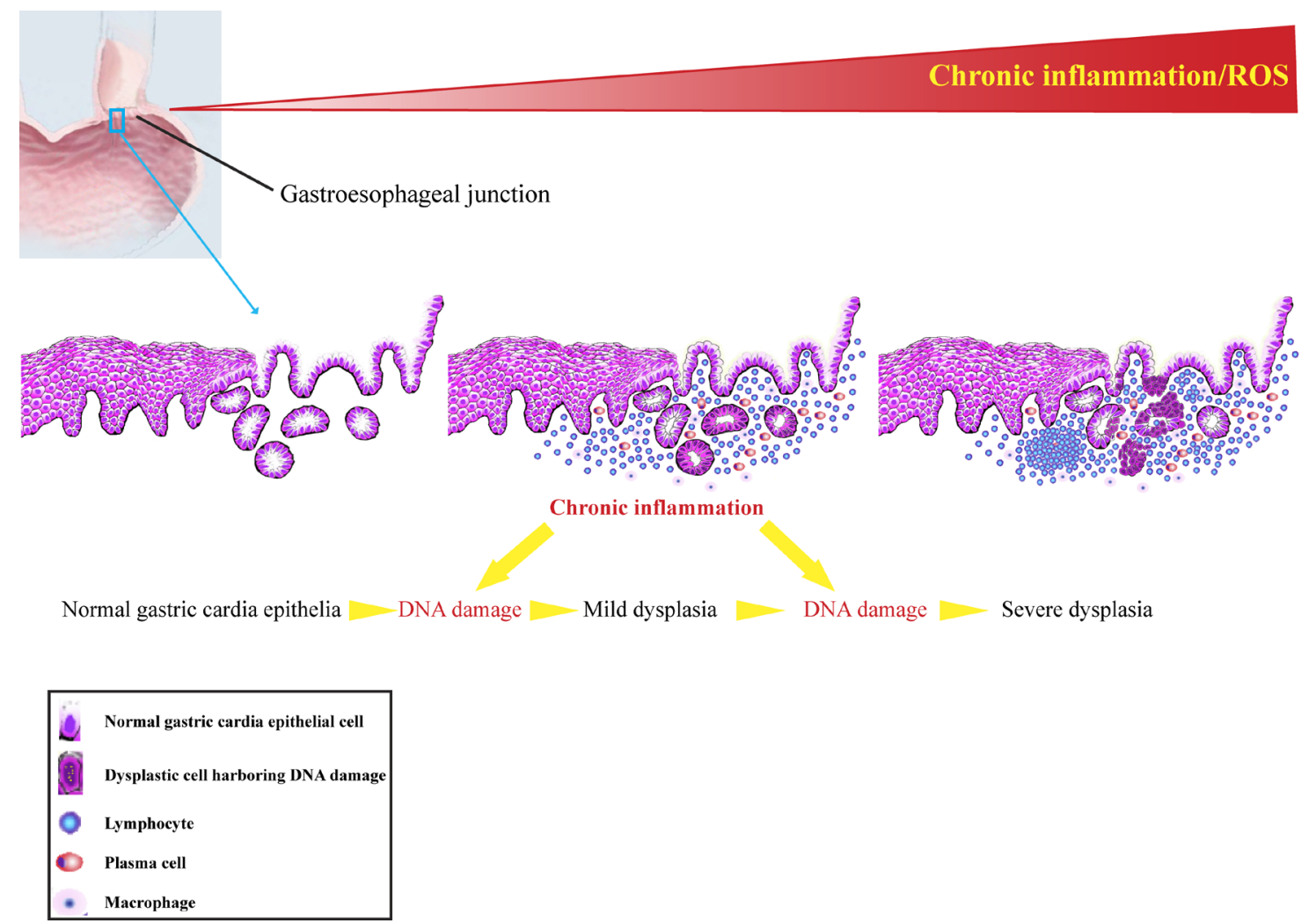

Figure 4: Schematic diagram of persistent chronic inflammation-related DNA damage response contributes to gastric cardia carcinogenesis. 
Subsequently, immunohistochemical studies revealed that the level of DNA damage response, evident as positive staining for $\gamma \mathrm{H} 2 \mathrm{AX}$ and phospho-ATM, was parallel with the severity of chronic inflammation, suggesting that the emergence of DNA damage response is likely to be induced by chronic inflammation. Furthermore, we showed that DDR levels were associated with premalignant lesions, indicating increased genomic instability in the multi-step process during gastric cardia carcinogenesis. These results provide pathological evidence in human gastric cardia tissues for the critical role of chronic inflammation-related DNA damage response in gastric cardia carcinogenesis.

Gastric cardia cancer ranks the second leading malignancy in Chaoshan region, a high-risk area for ESCC and GCC in southern China. Adenocarcinomas arising from the gastric cardia share the same histopathological features with esophageal adenocarcinoma (EAC), which is a common malignancy arises from metaplastic Barrett's esophagus caused by chronic exposure to acid and bile [23]. Gastro-esophageal reflux disease (GERD) is an established etiological factor of EAC [24], whereas, the etiology of GCC remains obscure. Our results revealed that a significantly higher proportion of severe inflammation was observed in dysplastic samples (80.9\%) compared to that in normal or simple hyperplastic tissues $(40.7 \%)(\mathrm{P}<0.001)$, pointing out that chronic inflammation may be an initiating factor of gastric cardia carcinogenesis in Chaoshan high-risk region. The existence of chronic inflammation in the gastric cardia may partly be elicited by Helicobacter pylori infection [25].

Inflammation is part of the human body's normal response to tissue damage inflicted by infections or other stimuli, when it becomes chronic, however, it can cause various pathologies including cancer [26-28]. Previous studies revealed that certain malignancies arise at sites affected by severe chronic inflammation [29, 30]. Actually, chronic inflammation might not contribute to carcinogenesis directly, since not all chronic inflammation ended up with cancer.

Tumor development is a multi-step process in which initially normal cells undergo a succession of intermediate stages in order to reach a fully malignant phenotype, functional changes of series of genes have occurred during this course [31-33]. Chronic inflammation might increase oxidative stress and damage to several biomolecules, including DNA, which may initiate or promote malignant transformation in the affected tissues. During chronic inflammatory process, immune cells, ranging in size, distribution and composition are recruited to the affected areas. These cells produce a variety of cytotoxic mediators, such as ROS and RNS, which were reported to bring about DNA damage [34]. DNA double-strand breaks (DSBs) are the most serious form of DNA damage and are difficult to repair accurately [35]. Accumulated DNA damage gives rise to mutagenesis and chromosomal rearrangements, contributing to carcinogenesis via genomic instability [36]. Phosphorylated H2AX is considered to be a reliable biomarker of DSBs [37].Our previous study showed that $\mathrm{CagA}+H$. pylori could induce strong DNA damage in human immortalized esophageal epithelial cells [38]. Moreover, increased levels of $\gamma \mathrm{H} 2 \mathrm{AX}$ were found in colonic mucosal specimens from patients with ulcerative colitis compared to control samples [39]. In this present study, our results demonstrated that a higher level of DNA damage response was detected in epithelia with severer inflammation, suggesting of a close association between chronic inflammation and DNA damage response. The elevated DNA damage response levels could be a result of increased production of RONS from infiltrating inflammatory cells. Additionally, we also detected higher level of DDR in tissues with different degrees of intraepithelial neoplasia compared to normal epithelia. Besides, several DNA damage response markers have been reported to be activated by chronic inflammatory stress [40]. The remarkable accumulation of DNA damage response in samples with intraepithelial neoplasia strongly indicates that unrepaired DNA damage in these precancerous tissues may be a main driving force of gastric cardia tumorigenesis. Hence, DNA damage response may be selected as a promising biomedical marker for indentifying potential carcinogenesis in individuals with chronic inflammation in the gastric cardia. Our results suggest a hypothesis (Figure 4) that increased gastric cardia epithelial cells harboring DDR in the milieu of chronic inflammation may precipitate carcinogenesis. As such, critical genes involving in cell proliferation and differentiation are probably affected due to the accumulated DNA damage, and prompt neoplastic transformation.

It has been reported that inflammation-related $\mathrm{NF}-\kappa \mathrm{B}$ pathway can activate mTOR pathway and thus regulate DDR [41], supporting our findings that higher level of DDR was frequently observed in inflamed tissues. Rapamycin, a clinically approved drug, was suggested to be potentially used to decrease DDR [42], this is also a promising therapeutic target for early intervention, for our data demonstrated that higher level of DDR presented in dysplastic tissues compared to the normal tissues.

In conclusion, this study showed that DNA damage response was detectable in gastric cardia mucosa with chronic inflammation. This DNA damage response correlated with the severity of chronic inflammation as well as the development of precancerous mucosal changes. Thus, we speculate that DNA damage response might serve as a critical link between chronic inflammation and gastric cardia malignancy through its high potential for causing DNA mutations, which is a main driving force of gastric cardia carcinogenesis. DNA damage response, therefore, might be considered as a promising diagnostic marker for identification of potential malignant transformation in gastric cardia biopsies at early stage, 
particularly in inflamed tissues. Given the relatively small sample size in our current study, future large-sample investigations should be performed to further evaluate the application value of DNA damage response in clinical setting.

\section{MATERIALS AND METHODS}

\section{Patients and sample collection}

A total of 50 paired tissue samples from GCC patients with no prior radio- or chemotherapy were collected from Cancer Hospital of Shantou University Medical College from January 2010 to December 2012. For each patient, tumor-surrounding non-malignant tissues (within $2 \mathrm{~cm}$ from tumor) and distant non-malignant tissues (more than $5 \mathrm{~cm}$ away from tumor) were analyzed. The total 100 samples were categorized as normal, lowgrade intraepithelial neoplasia (LGIN) and high-grade intraepithelial neoplasia (HGIN). All samples were fixed, dehydrated and paraffin embedded, and their pathological features and diagnoses were verified by two pathologists with hematoxylin and eosin staining. Baseline data of the 50 GCC patients and sample profile were shown in Table 1. This study was approved by Ethics Committee of Shantou University Medical College, and informed consents were obtained from patients or their families.

\section{Immunohistochemistry}

Paraffin-embedded samples were serially sectioned at $4 \mu \mathrm{m}$, mounted on gelatin-coated slides, dried at $60^{\circ} \mathrm{C}$ for $3 \mathrm{~h}$. The sections were deparaffinized in xylene, rehydrated in a descending series of ethanol solutions, and then treated with $3 \%$ hydrogen peroxide for $15 \mathrm{~min}$ to quench endogenous peroxidase activity. Antigen retrieval was performed by microwaving the sections immersed in citric acid buffer (10 mM, pH 6.0) for $20 \mathrm{~min}$, followed by $1 \mathrm{~h}$ incubation at room temperature. To block non-specific staining, we incubated sections with $10 \%$ normal goat serum. Next, primary antibodies for $\gamma \mathrm{H} 2 \mathrm{AX}$ and phosphoATM Ser1981 were added, and the slides were incubated at $4{ }^{\circ} \mathrm{C}$ overnight. After washing with PBS, corresponding secondary antibodies were added for incubation at $37^{\circ} \mathrm{C}$ for $30 \mathrm{~min}$ before reaction with diaminobenzidine and counterstaining with hematoxylin. Slides were then dehydrated and mounted. Positive controls were used for each of the antibodies (breast cancer tissues for $\gamma \mathrm{H} 2 \mathrm{AX}$ and phospho-ATM Ser1981), while in the negative controls the PBS was substituted for the primary antibody.

Images were captured using a Leica IM50 microscope at $\times 400$, and five different fields for each index were selected in each sample. The number of positive nuclei and total nuclei in images was then counted with the application of Image Pro Plus (IPP) 6.0 software, and the corresponding labeling index (percentage) was computed as positive nuclei/total nuclei $\times 100 \%$.

\section{Western blot}

Tissues were lysated in RIPA lysis buffer supplemented with protease inhibitor (Beyotime, China). Protein samples $(50 \mu \mathrm{g})$ were denatured, separated by SDS-polyacrylamide gel electrophoresis and transferred to PVDF membranes. After blocking in TBS with 5\% BSA, the membranes were incubated with primary antibodies against $\gamma \mathrm{H} 2 \mathrm{AX}(1: 1000$, Cell Signalling) and ACTIN (1:1000, Sigma Aldrich) overnight at $4^{\circ} \mathrm{C}$. After washing, the membranes were incubated with corresponding secondary antibodies at room temperature for $1 \mathrm{~h}$. The blotted membranes were washed and visualized using ECL reagents following the manufacturer's instructions.

\section{Statistical analysis}

All statistical analyses were performed using Statistical Package for Social Sciences (SPSS) 13.0 (SPSS Inc., Chicago, IL USA). One-way analysis of variance was used for labeling index analysis in multiple groups. The difference of labeling index between two groups was evaluated with $t$-test. A two-tailed $\mathrm{P} \leq 0.05$ was considered statistically significant. All reported P values were twosided.

\section{ACKNOWLEDGEMENTS}

This study was sponsored by National Natural Science Foundation of China (NSFC)-Guangdong Joint Fund Key Project (Certificate U1132004), grants of National Natural Science Foundation of China (Certificate 31171226), Guangdong International Cooperative Technical Innovation Platform (Certificate gihz1106), and Research Fund for the Doctoral Program of Higher Education of China (Certificate 20114402120006).

\section{Conflict of interest statement}

The authors declare no conflict of interest.

\section{REFERENCES}

1. Ferlay J, Shin HR, Bray F, Forman D, Mathers C and Parkin DM. Estimates of worldwide burden of cancer in 2008: GLOBOCAN 2008. International journal of cancer Journal international du cancer. 2010; 127(12):2893-2917.

2. Su M, Liu M, Tian DP, Li XY, Zhang GH, Yang HL, Fan X, Huang HH and Gao YX. Temporal trends of esophageal cancer during 1995-2004 in Nanao Island, an extremely 
high-risk area in China. European journal of epidemiology. 2007; 22(1):43-48.

3. He YT, Hou J, Chen ZF, Qiao CY, Song GH, Meng FS, Jin HX and Chen C. Trends in incidence of esophageal and gastric cardia cancer in high-risk areas in China. European journal of cancer prevention : the official journal of the European Cancer Prevention Organisation (ECP). 2008; 17(2):71-76.

4. Botterweck AA, Schouten LJ, Volovics A, Dorant E and van Den Brandt PA. Trends in incidence of adenocarcinoma of the oesophagus and gastric cardia in ten European countries. International journal of epidemiology. 2000; 29(4):645-654.

5. Vial M, Grande L and Pera M. Epidemiology of adenocarcinoma of the esophagus, gastric cardia, and upper gastric third. Recent results in cancer research Fortschritte der Krebsforschung Progres dans les recherches sur le cancer. 2010; 182:1-17.

6. Falk J, Carstens H, Lundell L and Albertsson M. Incidence of carcinoma of the oesophagus and gastric cardia. Changes over time and geographical differences. Acta oncologica (Stockholm, Sweden). 2007; 46(8):1070-1074.

7. McColl KE and Going JJ. Aetiology and classification of adenocarcinoma of the gastro-oesophageal junction/cardia. Gut. 2010; 59(3):282-284.

8. Aggarwal BB, Shishodia S, Sandur SK, Pandey MK and Sethi G. Inflammation and cancer: how hot is the link? Biochemical pharmacology. 2006; 72(11):1605-1621.

9. Rogler G. Chronic ulcerative colitis and colorectal cancer. Cancer letters. 2014; 345(2):235-241.

10. O'Byrne KJ and Dalgleish AG. Chronic immune activation and inflammation as the cause of malignancy. British journal of cancer. 2001; 85(4):473-483.

11. Balkwill $\mathrm{F}$ and Mantovani A. Inflammation and cancer: back to Virchow? Lancet. 2001; 357(9255):539-545.

12. Coussens LM and Werb Z. Inflammation and cancer. Nature. 2002; 420(6917):860-867.

13. Hussain SP and Harris CC. Inflammation and cancer: an ancient link with novel potentials. International journal of cancer Journal international du cancer. 2007; 121(11):23732380.

14. Rubin DT, Huo D, Kinnucan JA, Sedrak MS, McCullom NE, Bunnag AP, Raun-Royer EP, Cohen RD, Hanauer $\mathrm{SB}$, Hart J and Turner JR. Inflammation is an independent risk factor for colonic neoplasia in patients with ulcerative colitis: a case-control study. Clinical gastroenterology and hepatology : the official clinical practice journal of the American Gastroenterological Association. 2013; 11(12):1601-1608 e1601-1604

15. Atsumi T, Singh R, Sabharwal L, Bando H, Meng J, Arima Y, Yamada M, Harada M, Jiang JJ, Kamimura D, Ogura $\mathrm{H}$, Hirano $\mathrm{T}$ and Murakami M. Inflammation amplifier, a new paradigm in cancer biology. Cancer research. 2014; 74(1):8-14.
16. Ying S, Myers K, Bottomley S, Helleday T and Bryant HE. BRCA2-dependent homologous recombination is required for repair of Arsenite-induced replication lesions in mammalian cells. Nucleic acids research. 2009; 37(15):5105-5113.

17. Meira LB, Bugni JM, Green SL, Lee CW, Pang B, Borenshtein D, Rickman BH, Rogers AB, MoroskiErkul CA, McFaline JL, Schauer DB, Dedon PC, Fox JG and Samson LD. DNA damage induced by chronic inflammation contributes to colon carcinogenesis in mice. The Journal of clinical investigation. 2008; 118(7):25162525.

18. Ohnishi S, Ma N, Thanan R, Pinlaor S, Hammam O, Murata $\mathrm{M}$ and Kawanishi S. DNA damage in inflammation-related carcinogenesis and cancer stem cells. Oxidative medicine and cellular longevity. 2013; 2013:387014.

19. He H, Tian D, Guo J, Liu M, Chen Z, Hamdy FC, Helleday $\mathrm{T}, \mathrm{Su} \mathrm{M}$ and Ying S. DNA damage response in peritumoral regions of oesophageal cancer microenvironment. Carcinogenesis. 2013; 34(1):139-145.

20. Paull TT, Rogakou EP, Yamazaki V, Kirchgessner CU, Gellert $\mathrm{M}$ and Bonner WM. A critical role for histone $\mathrm{H} 2 \mathrm{AX}$ in recruitment of repair factors to nuclear foci after DNA damage. Current biology : CB. 2000; 10(15):886-895.

21. Gastric cancer and Helicobacter pylori: a combined analysis of 12 case control studies nested within prospective cohorts. Gut. 2001; 49(3):347-353.

22. Correa P. Human gastric carcinogenesis: a multistep and multifactorial process--First American Cancer Society Award Lecture on Cancer Epidemiology and Prevention. Cancer research. 1992; 52(24):6735-6740.

23. Quante M, Bhagat G, Abrams JA, Marache F, Good P, Lee MD, Lee Y, Friedman R, Asfaha S, Dubeykovskaya Z, Mahmood U, Figueiredo JL, Kitajewski J, Shawber C, Lightdale CJ, Rustgi AK, et al. Bile acid and inflammation activate gastric cardia stem cells in a mouse model of Barrett-like metaplasia. Cancer cell. 2012; 21(1):36-51.

24. Spechler SJ. Barrett esophagus and risk of esophageal cancer: a clinical review. JAMA : the journal of the American Medical Association. 2013; 310(6):627-636.

25. Wang Y, Liu S, Zhang Y, Bi C, Xiao Y, Lin R, Huang B, Tian D, Ying S and Su M. Helicobacter pylori infection and gastric cardia cancer in Chaoshan region. Microbes and infection / Institut Pasteur. 2014.

26. Vendramini-Costa DB and Carvalho JE. Molecular link mechanisms between inflammation and cancer. Current pharmaceutical design. 2012; 18(26):3831-3852.

27. Balkwill F, Charles KA and Mantovani A. Smoldering and polarized inflammation in the initiation and promotion of malignant disease. Cancer cell. 2005; 7(3):211-217.

28. Mantovani A, Allavena P, Sica A and Balkwill F. Cancerrelated inflammation. Nature. 2008; 454(7203):436-444.

29. Kundu JK and Surh YJ. Inflammation: gearing the journey to cancer. Mutation research. 2008; 659(1-2):15-30. 
30. Clevers H. At the crossroads of inflammation and cancer. Cell. 2004; 118(6):671-674.

31. Burnworth B, Arendt S, Muffler S, Steinkraus V, Brocker EB, Birek C, Hartschuh W, Jauch A and Boukamp P. The multi-step process of human skin carcinogenesis: a role for p53, cyclin D1, hTERT, p16, and TSP-1. European journal of cell biology. 2007; 86(11-12):763-780.

32. Fransen K, Klintenas M, Osterstrom A, Dimberg J, Monstein HJ and Soderkvist P. Mutation analysis of the BRAF, ARAF and RAF-1 genes in human colorectal adenocarcinomas. Carcinogenesis. 2004; 25(4):527-533.

33. Kim JW and Wang XW. Gene expression profiling of preneoplastic liver disease and liver cancer: a new era for improved early detection and treatment of these deadly diseases? Carcinogenesis. 2003; 24(3):363-369.

34. Roessner A, Kuester D, Malfertheiner P and SchneiderStock R. Oxidative stress in ulcerative colitis-associated carcinogenesis. Pathology, research and practice. 2008; 204(7):511-524.

35. Khanna KK and Jackson SP. DNA double-strand breaks: signaling, repair and the cancer connection. Nature genetics. 2001; 27(3):247-254.

36. Jackson SP. Sensing and repairing DNA double-strand breaks. Carcinogenesis. 2002; 23(5):687-696.

37. Geric M, Gajski G and Garaj-Vrhovac V. gamma$\mathrm{H} 2 \mathrm{AX}$ as a biomarker for DNA double-strand breaks in ecotoxicology. Ecotoxicology and environmental safety. 2014; 105:13-21.

38. Li WS, Tian DP, Guan XY, Yun H, Wang HT, Xiao Y, Bi $\mathrm{C}$, Ying $\mathrm{S}$ and $\mathrm{Su} \mathrm{M}$. Esophageal intraepithelial invasion of Helicobacter pylori correlates with atypical hyperplasia. International journal of cancer Journal international du cancer. 2013.

39. Chambers WM, Warren BF, Jewell DP and Mortensen NJ. Cancer surveillance in ulcerative colitis. The British journal of surgery. 2005; 92(8):928-936.

40. Blanco D, Vicent S, Fraga MF, Fernandez-Garcia I, Freire J, Lujambio A, Esteller M, Ortiz-de-Solorzano C, Pio $\mathrm{R}$, Lecanda $\mathrm{F}$ and Montuenga LM. Molecular analysis of a multistep lung cancer model induced by chronic inflammation reveals epigenetic regulation of p16 and activation of the DNA damage response pathway. Neoplasia (New York, NY). 2007; 9(10):840-852.

41. Guo F, Li J, Du W, Zhang S, O’Connor M, Thomas G, Kozma S, Zingarelli B, Pang Q and Zheng Y. mTOR regulates DNA damage response through NF-kappaBmediated FANCD2 pathway in hematopoietic cells. Leukemia. 2013; 27(10):2040-2046.

42. Bandhakavi S, Kim YM, Ro SH, Xie H, Onsongo G, Jun CB, Kim DH and Griffin TJ. Quantitative nuclear proteomics identifies mTOR regulation of DNA damage response. Molecular \& cellular proteomics : MCP. 2010; 9(2):403-414. 